\title{
Hybrid tandem and ovoids brachytherapy in locally advanced cervical cancer: impact of dose and tumor volume metrics on outcomes
}

\author{
Amanda Rivera, MD', Monica Wassel, BSc², Patrik N. Brodin, PhD',2, Ravindra Yaparpalvi, MSc', Christian Velten, MScl, \\ Rafi Kabarriti, MD', Madhur Garg, MD', Shalom Kalnicki, MD', Keyur J. Mehta, MD' \\ 'Department of Radiation Oncology, Montefiore Medical Center/Albert Einstein College of Medicine, New York, USA, ${ }^{2}$ Albert Einstein College \\ of Medicine, New York, USA
}

\begin{abstract}
Purpose: To report the impact of dose and tumor volume metrics at brachytherapy on outcomes for locally advanced cervical cancer treated with tandem and ovoids intracavitary/interstitial brachytherapy.

Material and methods: FIGO stage IB1-IIIB locally advanced cervical cancer treated with intracavitary/interstitial brachytherapy via a tandem and ovoids hybrid applicator were analyzed. Median high-risk clinical target volume (HR-CTV), rate of tumor volume reduction, $\mathrm{EQD}_{2} \mathrm{D}_{90}$, organ at risk doses, and outcomes were recorded. Univariable and multivariable Cox regression was applied for survival analysis, and logistic regression was used for toxicity analysis.

Results: Seventy-one patients were identified. Median follow-up was 24.9 months, with a 2-year local control of 83.6\%, loco-regional control of $72.0 \%$, and overall survival of $88.6 \%$. Median HR-CTV $\mathrm{D}_{90}$ was 87.4 Gy (IQR $=85.7-$ 90.2). Median HR-CTV $D_{90}>90 \mathrm{~Gy}_{10}$ showed a trend toward improved local control (LC) $(p=0.19)$. Median HR-CTV was $37.9 \mathrm{~cm}^{3}$, and median $\mathrm{V}_{100}$ was $86.5 \%$. A median HR-CTV of $\geq 40 \mathrm{~cm}^{3}$ demonstrated worse loco-regional control (LRC) $(p=0.018)$ and progression-free survival $(p=0.021)$. Two-year LC and LRC for stage IIB patients with a median HR-CTV $<40 \mathrm{~cm}^{3}$ were significantly improved as compared to $\geq 40 \mathrm{~cm}^{3}$ at $100 \%$ and $71.8 \%$, respectively $(p=0.019)$ and $100 \%$ and $56.5 \%$, respectively $(p=0.001)$. However, this trend was not statistically significant for stage IIIB patients. Higher percent per day reduction in HR-CTV during brachytherapy showed improved LRC $(p=0.045)$. Four percent of patients experienced acute grade 3 genitourinary toxicity, $1 \%$ late grade 3 genitourinary and $1 \%$ late grade 3 gastrointestinal toxicity.

Conclusions: Tandem and ovoids intracavitary/interstitial brachytherapy provides satisfactory outcomes with modest toxicity. Higher HR-CTV $\mathrm{D}_{90}$ coverage demonstrated a trend toward improved tumor control. Tumor volume based on median HR-CTV $\geq 40 \mathrm{~cm}^{3}$ at brachytherapy was prognostic for poor outcomes, even within initial FIGO stage groups warranting caution.

J Contemp Brachytherapy 2021; 13, 2: 158-166 DOI: https://doi.org/10.5114/jcb.2021.105283
\end{abstract}

Key words: cervical cancer, brachytherapy, hybrid device, tandem and ovoids.

\section{Purpose}

Locally advanced cervical cancer (LACC) remains a prevalent global disease, for which brachytherapy (BT) is of utmost importance for primary tumor control and overall survival [1]. The evolution of brachytherapy over the past two decades, driven by advances with image-guided brachytherapy (IGBT), for cervical cancer has resulted in excellent local control, survival, and toxicity outcomes [2]. Following the implementation of IGBT, there has been growing interest in the development of hybrid BT applicators that allow increased dose coverage of the parametria and pelvic sidewall for tu- mors not adequately treated with an intracavitary device [3]. Although hybrid intracavitary/interstitial (IC/IS) applicators are now in clinical use, there is limited large scale data outside of the EMBRACE studies surrounding clinical outcomes, as they relate to tumor and organs at risk (OARs) dose and tumor volume metrics. Specifically, there is a lack of data in patients treated with a tandem and ovoids hybrid brachytherapy approach. We sought to demonstrate the contemporary clinical outcomes of LACC patients treated with IC/IS BT using a hybrid tandem and ovoids device, and their association with tumor and OARs doses, and tumor volume metrics at brachytherapy. 


\section{Material and methods}

This retrospective analysis was performed following approval from our institutional review board. An institutional database was queried for LACC patients treated with concurrent external beam radiation therapy (EBRT) and chemotherapy followed by hybrid IC/IS BT from 2010-2017. EBRT was performed using computed tomography (CT)-based image-guided IMRT, with pelvic organ motion managed with an internal target volume (ITV)based approach. Patients were simulated with a full and empty bladder to account for motion of the vagina, cervix, and uterus. Gross tumor volume was delineated using $\mathrm{CT}$, positron emission tomography (PET/CT), and magnetic resonance imaging (MRI) fusion as per the preferred institutional standard. A clinical target volume (CTV)-cervix included a $0.5 \mathrm{~cm}$ expansion on the gross tumor volume. This was done on both full and empty bladder CT scans separately. A CTV including the CTV-cervix plus the vagina and uterus was generated on full and empty bladder scans separately, then combined into a single ITV-VCU (vagina cervix uterus). A $1.0 \mathrm{~cm}$ expansion was then applied to generate the PTV-VCU. A separate CTVLN (lymph node) of the pelvic and para-aortic (based on physician discretion) lymph nodes, and parametria was contoured with a $0.7 \mathrm{~cm}$ expansion to PTV-LN. Our institutional practice is to include para-aortic lymph nodes if there is an evidence of involved pelvic or para-aortic lymph nodes on imaging. Any gross lymphadenopathy had a $0.5 \mathrm{~cm}$ expansion to a CTV, which was included in the CTV-LN. The PTV-VCU and PTV-LN were combined for a total PTV, which was prescribed a dose of $45 \mathrm{~Gy}$ in $1.8 \mathrm{~Gy}$ fractions. A simultaneous integrated boost was given to the primary tumor and any gross lymphadenopathy to a dose of $50 \mathrm{~Gy}$ in $2 \mathrm{~Gy}$ fractions. Following the simultaneous integrated boost to $50 \mathrm{~Gy}$, any gross lymph nodes alone were sequentially boosted to a dose of 54-60 Gy, as they were not treated in the brachytherapy boost. The sequential lymph node boost dose was prescribed at the discretion of treating radiation oncologist on an individual basis.

\section{Brachytherapy}

Brachytherapy was administered in 6-8 Gy fractions over 3-5 insertions via a hybrid tandem and ovoids applicator utilizing an ${ }^{192}$ Ir afterloader high-dose-rate system. The Utrecht applicator (Elekta) was used for all cases in this series (Figure 1). Cervical Smit sleeve insertion was performed in the operating room prior to BT fractions, generally in the last weeks of EBRT. BT insertions were performed in the outpatient setting, with the use of a narcotic and anxiolytic regimen, and viscous lidocaine for pain control or conscious sedation. Tandem and ovoids were inserted followed by placement of a vaginal balloon posterior to the ovoids in the vaginal canal. In general, the tandem is inserted through the vaginal canal to pass through the cervical Smit sleeve, followed by placement of the ovoids with interstitial guide tubes in place. CT scan confirmation of the placement was used in all insertions, as well as, calculation of distance of interstitial needle insertion and adjustments. Placement of interstitial needles was decided upon for improved dosimetry based on parametrial or sidewall disease, or bulky disease at the time of brachytherapy. CT image-based contouring, treatment planning, and plan evaluation were performed according to the GEC ESTRO guidelines [4]. In patients who were treated prior to implementation of the updated image-guided adaptive BT (IGABT), high-risk clinical target volume (HR-CTV) definition, gross tumor volumes were excluded in the EQD 2 analysis for $\mathrm{HR}-\mathrm{CTV}$ and $\mathrm{D}_{90}$ calculations. During the study period, institutional practice was adapted to best incorporate the GEC ESTRO 3D image-based contouring guidelines. This was done on CT scan with the applicator in place, considering that MRI was not routinely available for each fraction. Radiology reports from CT, PET, and MRI (when available) were utilized to aid in delineation of the HR-CTV. Oncentra Treatment Planning System (version 4.3, Elekta) was utilized for all brachytherapy plans. In our institution, treatment planning procedure for hybrid Utrecht application begins with a customary tandem and ovoids treatment planning type process, with the dose normalized and optimized to applicator points and target HR-CTV points (resulting mostly in a pear-shaped dose distribution, except at the levels of interstitial needles). The resulting dose distributions are then reviewed on all slices (utilizing axial, sagittal, and coronal views) by the radiation oncologist and wherever necessary, the dose distributions are further refined by graphically changing local dwell times to modify isodose lines (Figure 2). This is done to achieve proper target coverage $\left(D_{90}\right)$, reduce hot spots, and to match patient anatomy while respecting OARs doses. A 6 or 7 Gy dose is prescribed to the $100 \%$ isodose line, while making every effort to limit the combined EBRT + BT OARs doses. Constraints include $\mathrm{D}_{2 \mathrm{cc}}<80$ Gy to the bladder, and $<70$ Gy to the rectum, sigmoid, and small bowel, equivalent dose in 2 Gy fractions $\left(E_{2} D_{2}\right)$. In general, the tandem and ovoids contribution is around $80 \%$ of the dose, with interstitial needles contributing to $20 \%$ of the dose.

Pre-brachytherapy MRI was utilized to assess treatment response to EBRT and to select patients for hybrid IC/IS BT, when available. The rate of reduction of tumor volume during BT was assessed by calculating the rate of change in HR-CTV from the CT acquired at each brachytherapy fraction.

Acute and late toxicities were graded according to CTCAE v. 5.0. Fistulas were classified once according to

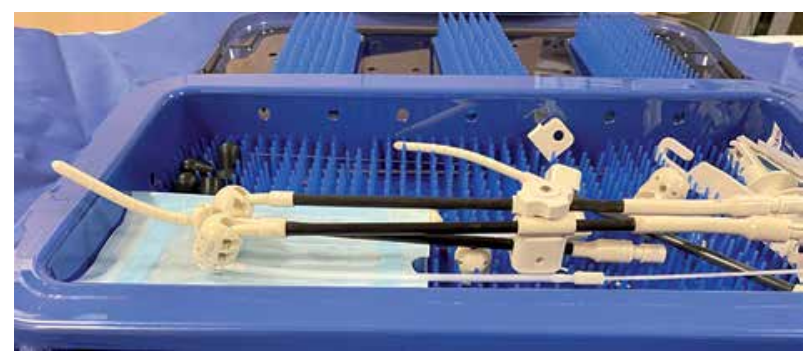

Fig. 1. Tandem and ovoids hybrid brachytherapy using Utrecht applicator. Shown are the tandem, ovoids, guiding tubes, and flexi-needles flushed with the ovoid surface 


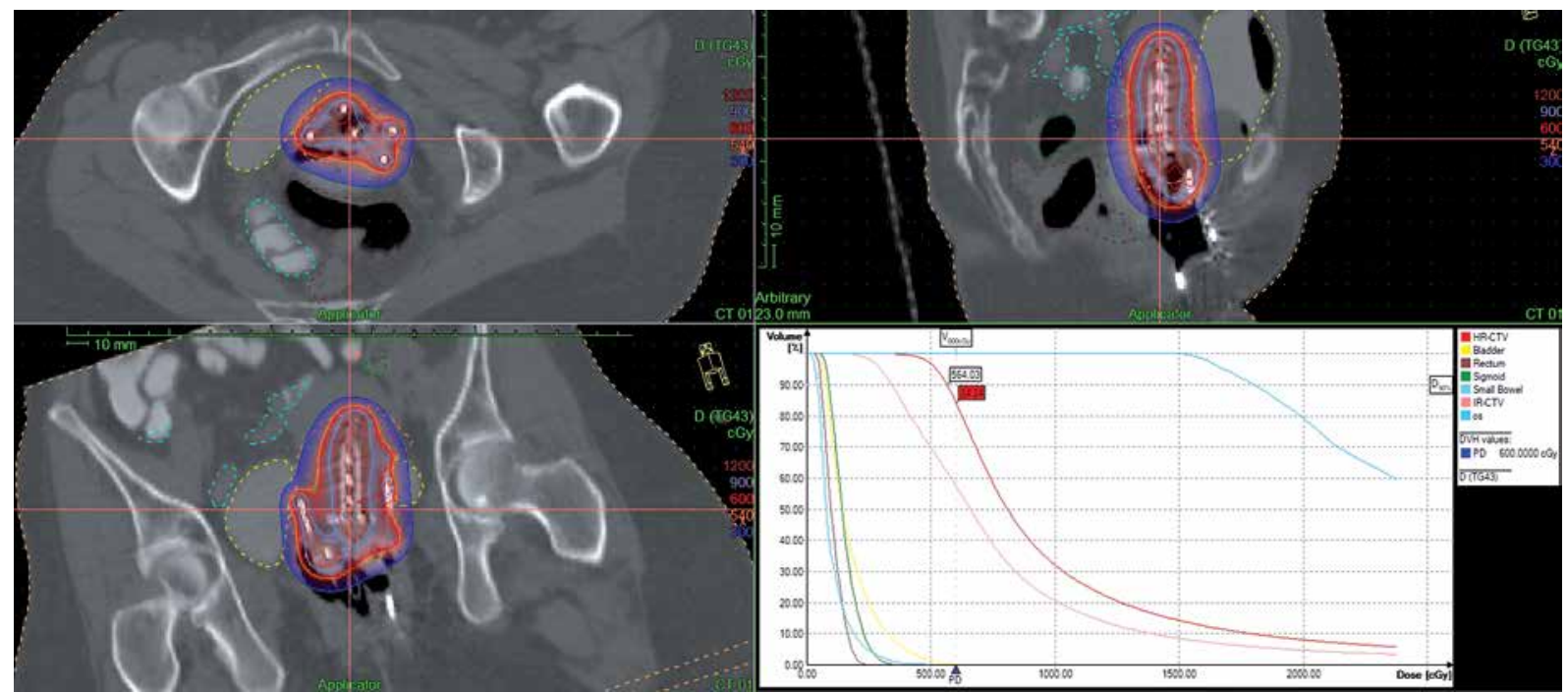

Fig. 2. Tandem and ovoids hybrid plan dose distribution and DVH. Top left - axial. Bottom left - coronal. Top right - sagittal. Bottom right - DVH analysis

the anatomical site involved. If a fistula was due to tumor involvement, this was not scored as treatment-induced toxicity.

Treatment parameters tabulated included HR-CTV $\mathrm{D}_{90}, \mathrm{~V}_{100}, 0.1 \mathrm{cc}, 1 \mathrm{cc}$, and $2 \mathrm{cc}$ doses to OARs, including the bladder, rectum, bowel, and sigmoid. The $\mathrm{D}_{90}$ and $\mathrm{D}_{0.1 \mathrm{cc}}, \mathrm{D}_{1 \mathrm{cc}}$ and $\mathrm{D}_{2 \mathrm{cc}}$ were recorded for each fraction and summed to a total BT-EQD ${ }_{2}$, which was then combined with the external beam $\mathrm{EQD}_{2}$ for the HR-CTV and OARs separately. These calculations were performed according to a linear quadratic formula assuming an $\alpha / \beta$ of 10 for tumor and 3 for OARs. Patient and tumor characteristics were analyzed, including FIGO staging (2009) [5], histology, pre-brachytherapy MRI tumor size, and the number of needles implanted. Median HR-CTV volume (mHRCTV) was also queried and analyzed for association with outcomes, as was the rate of reduction of tumor volume during BT.

\section{Statistical analyses}

Bivariate associations of clinical and dosimetric variables with median HR-CTV volume were assessed using Wilcoxon rank-sum tests for continuous variables and $\chi^{2}$ tests for categorical variables. Kaplan-Meier method was used to estimate local control (LC), loco-regional control (LRC), distant control (DC), and overall survival (OS), with time-to-event data censored at the corresponding event or time of last contact within our health system. Cox proportional hazards regression was applied to analyze association with survival outcomes, and logistic regression was used to evaluate association with toxicity. All statistical analyses were performed in STATA v.14 (StataCorp, College Station, TX, USA).

\section{Results}

Seventy-one patients with locally advanced cervical tumors treated with hybrid tandem and ovoids IC/IS BT following definitive chemo-radiation were identified
(Table 1). The median follow-up was 24.9 months, with a two-year LC of $83.6 \%$. Two-year actuarial DC was $68 \%$, LRC $72.0 \%$, and OS was $88.6 \%$. We observed $23(32.4 \%)$ distant failures (DF), 12 (16.9\%) local failures (LF), and $13(18.3 \%)$ loco-regional failures (LRF). Six LF were identified as cervix only failures, without LRF or DF. Thirteen DF had no associated loco-regional or local failures. Eight patients with LRF had failure in the para-aortic lymph nodes, which were treated in the initial plan with extended field RT. Twelve patients with DF failed in the lungs, followed by the supraclavicular LNs (5) and the liver (4).

The mHR-CTV $\mathrm{D}_{90} \mathrm{EQD}_{2}$ was $87.4 \mathrm{~Gy}(\mathrm{IQR}=85.7$ 90.2) for all patients. FIGO IIB and IIIB patients had a mHR-CTV $\mathrm{D}_{90} \mathrm{EQD}_{2}$ of $87.5 \mathrm{~Gy}(\mathrm{IQR}=86.3-91.2)$ and 86.8 Gy (IQR = 83.9-89.6), respectively. The median number of needles placed per patient was 2 (range, 1-6). Forty-six percent of FIGO IIIB patients and twenty-nine percent of FIGO IIB patients had a median number of needles per fraction $>2$. The mean, median (range) needles per brachytherapy course for FIGO IB1/IB2, IIB, and IIIB were 8.8, 8 (range, 5-14); 10.2, 10 (range, 4-28); 11.6, 10.5 (range, 3-20), respectively. Pre-brachytherapy MRI reported tumor size was available for 26 patients, with a median of $2.2 \mathrm{~cm}$ in diameter. In total, thirty-five patients had a pre-brachytherapy MRI completed.

The mHR-CTV volume for all patients was $37.9 \mathrm{~cm}^{3}$ and the median $\mathrm{V}_{100}$ was $86.5 \%$. The median $\mathrm{mHR}-\mathrm{CTV}$ in patients with pre-brachytherapy MRI was $37.4 \mathrm{~cm}^{3}$ (range, 23.8-100.1), and for those without pre-brachytherapy MRI was $42.0 \mathrm{~cm}^{3}$ (range, 16.4-108.5). However, there was no difference in local control between these two groups (HR $=0.95, p=0.93$ ). A mHR-CTV volume of $<40 \mathrm{~cm}^{3}$ was significantly associated with improved LRC (HR $=3.52,95 \%$ CI: 1.24-9.99, $p=0.018$, and improved progression-free survival (PFS) $(\mathrm{HR}=2.38,95 \% \mathrm{CI}: 1.14-4.95, p=0.021)$ on univariable analysis (Table 2 ). This remained statistically significant on multivariable analysis (Table 3).

Stage IIB patients with a mHR-CTV $<40 \mathrm{~cm}^{3}$ had significantly improved two-year LC as compared to 
Table 1. Patient and tumor characteristics

\begin{tabular}{|c|c|c|c|c|}
\hline & $\begin{array}{l}\text { All patients } \\
\quad(n=71)\end{array}$ & $\begin{array}{l}\text { Median HR-CTV } \\
<40 \mathrm{~cm}^{3}(n=38)\end{array}$ & $\begin{array}{l}\text { Median HR-CTV } \\
\geq 40 \mathrm{~cm}^{3}(n=28)\end{array}$ & $p$-value \\
\hline Age (years), median (range) & $55(32.7-90.8)$ & $55(32.7-90.8)$ & $54(38.2-88.9)$ & 0.96 \\
\hline \multicolumn{5}{|l|}{ Stage, $n(\%)$} \\
\hline IB1/IB2 & $5(7)$ & $1(3)$ & $2(7)$ & \multirow[t]{3}{*}{0.68} \\
\hline$\| \mathrm{B}$ & $38(54)$ & $21(55)$ & $15(54)$ & \\
\hline$\| \mathrm{IIB}$ & $28(39)$ & $16(42)$ & $11(39)$ & \\
\hline \multicolumn{5}{|l|}{ Pathology, $n(\%)$} \\
\hline SCC & $51(72)$ & $26(68)$ & $21(75)$ & \multirow[t]{4}{*}{0.76} \\
\hline Adenocarcinoma & $15(21)$ & $9(24)$ & $5(18)$ & \\
\hline Adenosquamous & $4(6)$ & $2(5)$ & $2(7)$ & \\
\hline Poorly differentiated & $1(1)$ & $1(3)$ & $0(0)$ & \\
\hline Median needles per fraction, median (range) & $2(1-6)$ & $2(1-4)$ & $3(1-4)$ & 0.001 \\
\hline Total needles, median (range) & $10(3-28)$ & $12(5-20)$ & $14(8-28)$ & 0.011 \\
\hline \multicolumn{5}{|l|}{ Concurrent chemotherapy, $n$ (\%) } \\
\hline Yes & $69(99)$ & $37(100)$ & $27(96)$ & \multirow[t]{2}{*}{0.25} \\
\hline No & $1(1)$ & $0(0)$ & $1(4)$ & \\
\hline HR-CTV $D_{90} E D_{2}\left(G y_{10}\right)$, median (range) & $87.43(73.29-99.10)$ & $87.89(81.58-99.10)$ & $87.10(73.29-98.70)$ & 0.22 \\
\hline Median HR-CTV, volume $\mathrm{cm}^{3}$ (range) & $37.87(16.44-108.45)$ & $31.20(16.44-39.80)$ & $52.48(40.33-108.45)$ & $<0.001$ \\
\hline Median IR-CTV, volume $\mathrm{cm}^{3}$ (range) & $119.39(65.48-235.44)$ & $105.19(65.48-130.12)$ & $159.02(110.06-235.44)$ & $<0.001$ \\
\hline Median $V_{100}$ (range) & 86.48 (71.77-99.03) & 87.08 (71.77-99.03) & 85.94 (71.90-96.74) & 0.84 \\
\hline $\mathrm{EQD}_{2}$ bladder, $0.1 \mathrm{cc}$, median (range) & $102.85(88.55-135.50)$ & $101.16(88.55-135.50)$ & $105.95(89.15-132.72)$ & 0.034 \\
\hline $\mathrm{EQD}_{2}$ bladder, $2 \mathrm{cc}$, median (range) & 88.27 (76.50-99.71) & 86.36 (76.50-99.67) & 89.09 (78.66-99.71) & 0.071 \\
\hline $\mathrm{EQD}_{2}$ bowel, $0.1 \mathrm{cc}$, median (range) & $74.86(53.23-110.80)$ & $76.90(53.60-110.80)$ & $67.04(53.23-104.65)$ & 0.33 \\
\hline $\mathrm{EQD}_{2}$ bowel, $2 \mathrm{cc}$, median (range) & $65.32(52.09-86.14)$ & $66.42(52.40-86.14)$ & $61.44(52.09-86.00)$ & 0.60 \\
\hline $\mathrm{EQD}_{2}$ rectum, $0.1 \mathrm{cc}$, median (range) & 83.15 (61.68-99.04) & 79.47 (61.68-99.04) & 87.96 (62.76-96.21) & 0.14 \\
\hline $\mathrm{EQD}_{2}$ rectum, $2 \mathrm{cc}$, median (range) & $69.96(57.99-82.99)$ & $68.58(57.99-81.42)$ & 73.65 (58.85-82.99) & 0.049 \\
\hline EQD 2 sigmoid, $0.1 \mathrm{cc}$, median (range) & $85.22(58.26-119.09)$ & $79.49(58.26-105.48)$ & $92.23(62.29-119.09)$ & 0.002 \\
\hline $\mathrm{EQD}_{2}$ sigmoid, $2 \mathrm{cc}$, median (range) & $70.40(54.88-90.32)$ & $67.75(54.88-81.14)$ & $75.28(57.80-90.32)$ & 0.004 \\
\hline $\begin{array}{l}\text { Reduction in HR-CTV during HDR course } \\
\text { (\%/day), median (range) }\end{array}$ & $0.40(-3.84-8.68)$ & $0.63(-3.84-8.68)$ & $0.13(-3.27-2.85)$ & 0.18 \\
\hline
\end{tabular}

SCC-squamous cell carcinoma, HR-CTV-high-risk clinical target volume, HDR - high-dose-rate, IR-CTV-intermediate risk clinical target volume

a mHR-CTV of $\geq 40 \mathrm{~cm}^{3}$ at $100 \%$ and $71.8 \%$, respectively $(p=0.019)$. Stage IIIB patients also demonstrated higher two-year LC, with a mHR-CTV of $<40 \mathrm{~cm}^{3}(85.1 \%$ vs. $70.7 \%)(p=0.41)$. Two-year LRC for stage IIB patients with a mHR-CTV of $<40 \mathrm{~cm}^{3}$ was significantly higher than those with a mHR-CTV $\geq 40 \mathrm{~cm}^{3}$ at $100 \%$ and $56.5 \%$, respectively $(p=0.001)$. However, two-year LRC for stage IIIB patients did not demonstrate this $(66.7 \%$ vs. $60.6 \%)$ $(p=0.76)$. Two-year PFS rates for stage IIB patients with a tumor volume of $<40$ and $\geq 40 \mathrm{~cm}^{3}$ were $77.8 \%$ and $45.7 \%$, respectively ( $p=0.051)$, and for stage IIIB patients were $66.7 \%$ and $40.4 \%$, respectively $(p=0.18)$. The median tumor volume for stage IIB and IIIB was $37.9 \mathrm{~cm}^{3}$ (range, 21.8-74.3) and $35.5 \mathrm{~cm}^{3}$ (range, 16.4-108.5), respectively.

A HR-CTV $\mathrm{D}_{90} \mathrm{EQD}_{2}>90 \mathrm{~Gy}_{10}$ was associated with improved LC; however, it did not reach statistical significance $(\mathrm{HR}=0.25, p=0.19)$ (Figure 3). Total treatment package time $>56$ days was significantly associated with worse loco-regional control $(p=0.047)$. A larger percent per day reduction in HR-CTV during BT was significantly associated with improved LRC $(\mathrm{HR}=0.73$, range, 0.54-0.99, $p=0.045$ ) (Table 3). 
Table 2. Univariable analysis-clinical characteristics, dose and tumor volume metrics

\begin{tabular}{|c|c|c|c|c|c|c|}
\hline & \multicolumn{2}{|c|}{ LRC } & \multicolumn{2}{|c|}{ PFS } & \multicolumn{2}{|c|}{ OS } \\
\hline & $\mathrm{HR}(95 \% \mathrm{Cl})$ & $p$-value & $\mathrm{HR}(95 \% \mathrm{Cl})$ & $p$-value & $\mathrm{HR}(95 \% \mathrm{Cl})$ & $p$-value \\
\hline $\begin{array}{l}\text { Median HR-CTV vol } \\
\left(<40 \mathrm{~cm}^{3} \text { vs. } \geq 40 \mathrm{~cm}^{3}\right)\end{array}$ & $\begin{array}{c}3.52 \\
(1.24,9.99)\end{array}$ & 0.018 & $\begin{array}{c}2.38 \\
(1.14,4.95)\end{array}$ & 0.021 & $\begin{array}{c}1.96 \\
(0.63,6.12)\end{array}$ & 0.25 \\
\hline Median HR-CTV vol $\left(\right.$ per $\left.\mathrm{cm}^{3}\right)$ & $\begin{array}{c}1.02 \\
(1.00,1.04) \\
\end{array}$ & 0.048 & $\begin{array}{c}1.01 \\
(1.00,1.03) \\
\end{array}$ & 0.10 & $\begin{array}{c}0.99 \\
(0.96,1.02) \\
\end{array}$ & 0.61 \\
\hline Age (per year) & $\begin{array}{c}0.99 \\
(0.95,1.03)\end{array}$ & 0.55 & $\begin{array}{c}0.99 \\
(0.97,1.02)\end{array}$ & 0.71 & $\begin{array}{c}0.96 \\
(0.91,1.01)\end{array}$ & 0.13 \\
\hline Stage (IIIB vs. IIB) & $\begin{array}{c}1.90 \\
(0.76,4.72)\end{array}$ & 0.17 & $\begin{array}{c}1.01 \\
(0.50,2.06)\end{array}$ & 0.97 & $\begin{array}{c}1.06 \\
(0.37,3.08)\end{array}$ & 0.91 \\
\hline $\begin{array}{l}\text { Histology (adeno or adeno/SCC } \\
\text { vs. SCC) }\end{array}$ & $\begin{array}{c}1.39 \\
(0.53,3.65)\end{array}$ & 0.51 & $\begin{array}{c}1.55 \\
(0.73,3.31) \\
\end{array}$ & 0.26 & $\begin{array}{c}1.56 \\
(0.47,5.22)\end{array}$ & 0.47 \\
\hline $\begin{array}{l}\text { Median number of needles per } \mathrm{fx} \\
\text { (per needle) }\end{array}$ & $\begin{array}{c}1.11 \\
(0.75,1.63)\end{array}$ & 0.60 & $\begin{array}{c}1.16 \\
(0.84,1.62)\end{array}$ & 0.35 & $\begin{array}{c}0.84 \\
(0.52,1.36)\end{array}$ & 0.48 \\
\hline Total number of needles (per needle) & $\begin{array}{c}1.01 \\
(0.92,1.10) \\
\end{array}$ & 0.85 & $\begin{array}{c}1.04 \\
(0.97,1.12) \\
\end{array}$ & 0.31 & $\begin{array}{c}0.96 \\
(0.86,1.07) \\
\end{array}$ & 0.45 \\
\hline $\mathrm{EQD}_{2} \mathrm{HDR}+\mathrm{EBRT}$ (per Gy) & $\begin{array}{c}1.00 \\
(0.90,1.10)\end{array}$ & 0.96 & $\begin{array}{c}1.02 \\
(0.94,1.10)\end{array}$ & 0.66 & $\begin{array}{c}0.99 \\
(0.88,1.12) \\
\end{array}$ & 0.90 \\
\hline $\begin{array}{l}\mathrm{EQD}_{2} \mathrm{HDR}+\mathrm{EBRT} \\
(\leq 90 \text { Gy vs. }>90 \mathrm{~Gy})\end{array}$ & $\begin{array}{c}0.51 \\
(0.15,1.79)\end{array}$ & 0.30 & $\begin{array}{c}1.01 \\
(0.45,2.29)\end{array}$ & 0.98 & $\begin{array}{c}1.04 \\
(0.28,3.88)\end{array}$ & 0.95 \\
\hline Median IR-CTV vol $\left(\right.$ per $\left.\mathrm{cm}^{3}\right)$ & $\begin{array}{c}1.01 \\
(1.0,1.02) \\
\end{array}$ & 0.082 & $\begin{array}{c}1.01 \\
(1.00,1.01) \\
\end{array}$ & 0.12 & $\begin{array}{c}1.00 \\
(0.99,1.01) \\
\end{array}$ & 0.82 \\
\hline $\begin{array}{l}\text { Change in HR-CTV vol during HDR } \\
\text { course (increase vs. decrease) }\end{array}$ & $\begin{array}{c}3.01 \\
(1.14,7.94)\end{array}$ & 0.026 & $\begin{array}{c}1.15 \\
(0.55,2.42)\end{array}$ & 0.72 & $\begin{array}{c}0.35 \\
(0.08,1.60)\end{array}$ & 0.18 \\
\hline $\begin{array}{l}\text { Reduction in HR-CTV vol during HDR } \\
\text { course (per \%/day) }\end{array}$ & $\begin{array}{c}0.77 \\
(0.58,1.02) \\
\end{array}$ & 0.069 & $\begin{array}{c}0.91 \\
(0.75,1.10) \\
\end{array}$ & 0.32 & $\begin{array}{c}0.97 \\
(0.76,1.25) \\
\end{array}$ & 0.83 \\
\hline Median HR-CTV V ${ }_{100}($ per \%) & $\begin{array}{c}1.01 \\
(0.94,1.10)\end{array}$ & 0.76 & $\begin{array}{c}1.03 \\
(0.97,1.09)\end{array}$ & 0.41 & $\begin{array}{c}1.01 \\
(0.93,1.10)\end{array}$ & 0.77 \\
\hline $\begin{array}{l}\text { Total treatment time } \\
(\leq 56 \text { days vs. }>56 \text { days) }\end{array}$ & $\begin{array}{c}4.43 \\
(1.02,19.2)\end{array}$ & 0.047 & $\begin{array}{c}2.15 \\
(0.88,5.26)\end{array}$ & 0.10 & $\begin{array}{c}1.04 \\
(0.28,3.92)\end{array}$ & 0.95 \\
\hline $\begin{array}{l}\text { No. of HDR fractions } \\
(<5 \mathrm{fx} \text { vs. } 5 \mathrm{fx})\end{array}$ & $\begin{array}{c}1.21 \\
(0.43,3.35)\end{array}$ & 0.72 & $\begin{array}{c}0.85 \\
(0.35,2.06)\end{array}$ & 0.72 & $\begin{array}{c}0.74 \\
(0.16,3.38)\end{array}$ & 0.70 \\
\hline
\end{tabular}

LRC - loco-regional control, PFS - progression-free survival, OS - overall survival, SCC - squamous cell carcinoma, HR-CTV - high-risk clinical target volume, $H D R$ - high-dose-rate, EBRT - external beam radiation therapy, IR-CTV - intermediate risk clinical target volume

The median bladder $0.1 \mathrm{cc}$ and $2 \mathrm{cc} \mathrm{EQD}_{2}$ doses were 102.9 Gy and $88.3 \mathrm{~Gy}$, respectively (Table 4). The median rectum $0.1 \mathrm{cc}$ and $2 \mathrm{cc} \mathrm{EQD}_{2}$ doses were $83.2 \mathrm{~Gy}$ and $70.0 \mathrm{~Gy}$, respectively. Notably, the sigmoid $0.1 \mathrm{cc} \mathrm{EQD}_{2}$ dose was associated with late gastrointestinal (GI) toxicity, with a HR of 1.06 (95\% CI: 1.01-1.10, $p=0.015)$ (Table 5). Bladder $\mathrm{EQD}_{2}$ doses were not associated with late genitourinary $(\mathrm{GU})$ toxicity. Neither rectum nor bowel $\mathrm{EQD}_{2}$ doses were associated with late GI toxicity.

Acute grade $2-3$ GU toxicities occurred in $12.7 \%$ of the patients. There were no acute grade 4 or higher GU toxicities. Acute grade 2 GI toxicity occurred in $24 \%$ of the patients. There were no acute grade 3 or higher GI toxicities. One patient experienced a grade 2 vaginal hemorrhage, which occurred prior to starting EBRT, but recurred due to non-compliance with EBRT course. One patient experienced a grade $3 \mathrm{GU}$ toxicity secondary to urosepsis during BT, which occurred due to a delay in percutaneous nephrostomy exchange. Another patient experienced an acute grade 3 vaginal hemorrhage, which occurred during the first week of EBRT.
Twelve percent of the patients experienced late grade 2 GU toxicity, and $1 \%$ suffered from late grade $3 \mathrm{GU}$ toxicity. Twenty-one percent of the patients experienced late grade 2 GI toxicity, and $1 \%$ late grade 3 GI toxicity. There were no grade 5 toxicities. There were two treatment related fistulas, 1 rectovaginal fistula, and 1 vesicovaginal fistula. A rectovaginal fistula occurred in a patient with initial FIGO IIIB disease and rectal 2 cc EQD Ef $_{2} .4$ Gy. A vesicovaginal fistula occurred in a patient with initial FIGO IIIB disease and bladder 2 cc EQD 2 of 95.9 Gy.

\section{Discussion}

We showed satisfactory clinical outcomes and modest toxicity in this large single-institution cohort of 71 patients, treated with hybrid tandem and ovoids IC/IS BT. Several applicators capable of both intracavitary and interstitial implantation have emerged, including tandem and ring, and tandem and ovoids-based designs. The Vienna applicator (Elekta, Veenendaal, The Netherlands; Varian, Palo Alto, USA) is a variation of a ring applica- 
Table 3. Multivariable analysis - clinical characteristics, dose and tumor volume metrics

\begin{tabular}{|c|c|c|c|c|c|c|}
\hline & \multicolumn{2}{|c|}{ LRC } & \multicolumn{2}{|c|}{ PFS } & \multicolumn{2}{|c|}{ OS } \\
\hline & $\mathrm{HR}(95 \% \mathrm{Cl})$ & $p$-value & $\mathrm{HR}(95 \% \mathrm{Cl})$ & $p$-value & $\mathrm{HR}(95 \% \mathrm{Cl})$ & $p$-value \\
\hline $\begin{array}{l}\text { Median HR-CTV volume } \\
\left(<40 \mathrm{~cm}^{3} \text { vs. } \geq 40 \mathrm{~cm}^{3}\right)\end{array}$ & $\begin{array}{c}3.86 \\
(1.35,11.03) \\
\end{array}$ & 0.012 & $\begin{array}{c}2.22 \\
(1.05,4.70)\end{array}$ & 0.038 & $\begin{array}{c}1.97 \\
(0.61,6.35)\end{array}$ & 0.26 \\
\hline Stage (IIIB vs. IIB) & $\begin{array}{c}2.65 \\
(0.98,7.14)\end{array}$ & 0.054 & $\begin{array}{c}1.30 \\
(0.60,2.77)\end{array}$ & 0.51 & $\begin{array}{c}0.95 \\
(0.30,3.03)\end{array}$ & 0.94 \\
\hline $\begin{array}{l}\text { Reduction in HR-CTV volume during } \\
\text { HDR course (per \%/day) }\end{array}$ & $\begin{array}{c}0.73 \\
(0.54,0.99)\end{array}$ & 0.045 & $\begin{array}{c}0.90 \\
(0.73,1.10)\end{array}$ & 0.30 & $\begin{array}{c}0.99 \\
(0.76,1.29)\end{array}$ & 0.94 \\
\hline Age (per year) & - & - & - & - & $\begin{array}{c}0.96 \\
(0.92,1.02)\end{array}$ & 0.19 \\
\hline
\end{tabular}

LRC - loco-regional control, PFS - progression-free survival, OS - overall survival, HR-CTV-high-risk clinical target volume, HDR - high-dose-rate

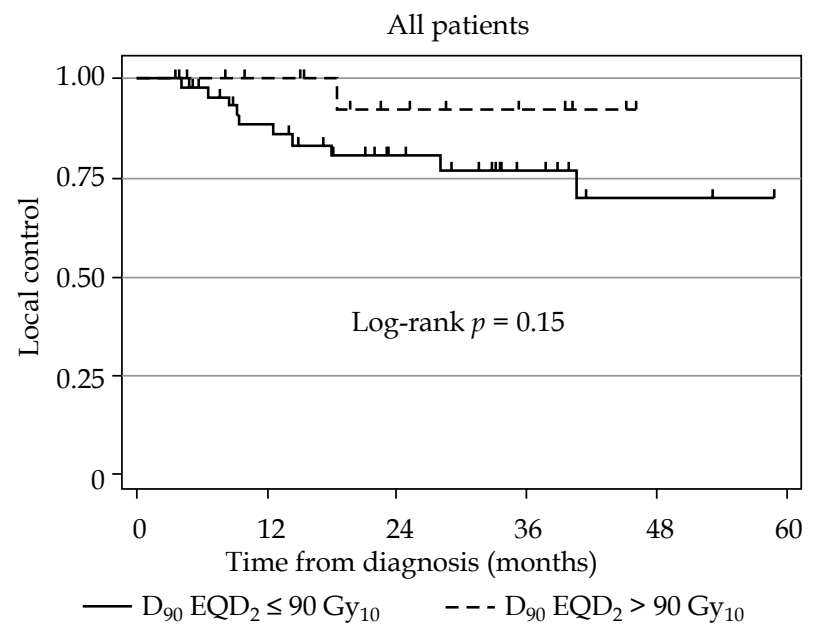

Number at risk

$\begin{array}{lllcccc}- & 48 & 36 & 24 & 14 & 9 & 7 \\ -- & 18 & 15 & 9 & 5 & 1 & 1\end{array}$

Patients with median HR-CTV $\geq 40 \mathrm{~cm}^{3}$

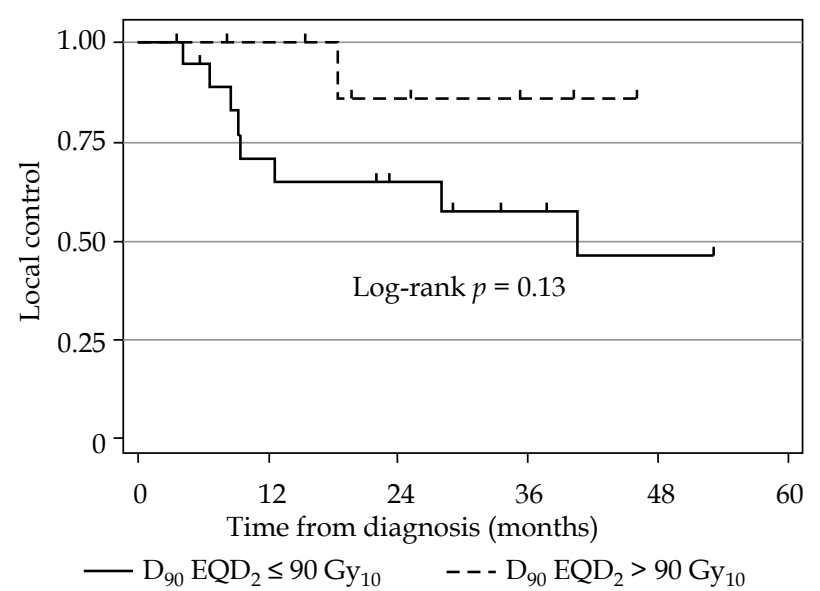

Number at risk

$\begin{array}{ccccccc}- & 19 & 12 & 9 & 6 & 4 & 3 \\ -- & 9 & 8 & 5 & 3 & 1 & 1\end{array}$

tor modified with interstitial needle positions available through the ring [6]. A subsequent, recently described adjustment to this applicator allows for oblique needle placement through the ring of the initial Vienna applicator, named "the Vienna-II" [7]. The Utrecht applicator

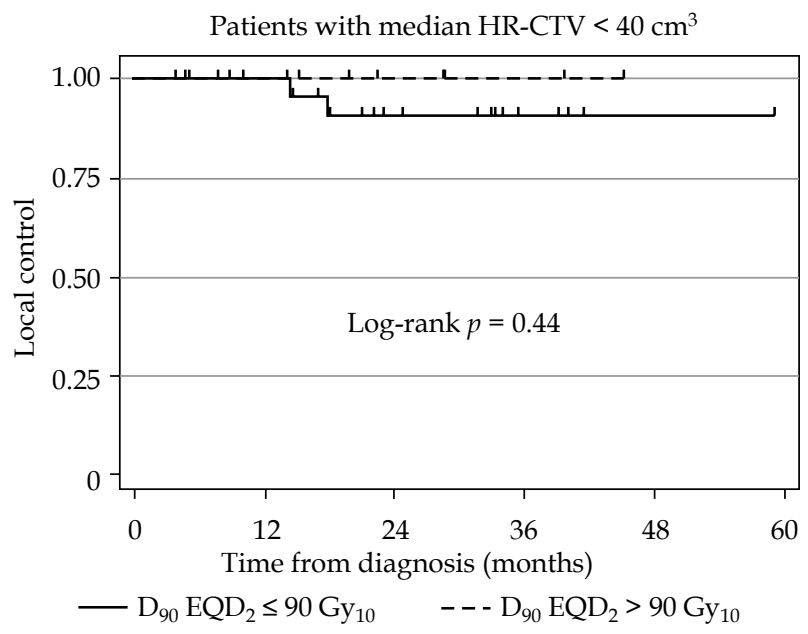

Number at risk

$\begin{array}{ccccccc}- & 29 & 24 & 15 & 8 & 5 & 4 \\ --- & 9 & 7 & 4 & 2 & 0 & 0\end{array}$

Fig. 3. HR-CTV $\mathrm{D}_{90}$ and local control. Higher HR-CTV $\mathrm{D}_{90}$ demonstrates a trend towards improved local control in patients with large (median HR-CTV volume $\geq 40 \mathrm{~cm}^{3}$ ) and small tumors $\left(<40 \mathrm{~cm}^{3}\right)$

(Elekta) is an adaptation of the tandem and ovoids applicator, with interstitial parallel needle placement available via the channels in the ovoids [6]. In this study population, patients were treated with a tandem and ovoids applicator with interstitial needles delivered through the 
Table 4. Organs at risk doses from brachytherapy and external beam therapy combined, presented as equivalent dose in 2 Gy fractions based on $\alpha / \beta=3$

\begin{tabular}{lc} 
Organ at risk dose & Median (range), $\left(\mathrm{Gy}_{3}\right)$ \\
\hline$E Q D_{2}$ bladder $0.1 \mathrm{~cm}^{3}$ & $102.9(88.5-135.5)$ \\
\hline$E Q D_{2}$ bladder $2 \mathrm{~cm}^{3}$ & $88.3(76.5-99.7)$ \\
\hline$E Q D_{2}$ bowel $0.1 \mathrm{~cm}^{3}$ & $74.9(53.2-110.8)$ \\
\hline$E Q D_{2}$ bowel $2 \mathrm{~cm}^{3}$ & $65.3(52.1-86.1)$ \\
\hline $\mathrm{EQD}_{2}$ rectum $0.1 \mathrm{~cm}^{3}$ & $83.2(61.7-99.0)$ \\
\hline $\mathrm{EQD}_{2}$ rectum $2 \mathrm{~cm}^{3}$ & $70.0(58.0-83.0)$ \\
\hline $\mathrm{EQD}_{2}$ sigmoid $0.1 \mathrm{~cm}^{3}$ & $85.2(58.3-119.1)$ \\
\hline $\mathrm{EQD}_{2}$ sigmoid $2 \mathrm{~cm}^{3}$ & $70.4(54.9-90.3)$
\end{tabular}

ovoid channels, using an image-guided BT approach. $3 \mathrm{D}$ contouring and treatment planning were performed, according to the published GEC-ESTRO guidelines, which define the HR-CTV or CTV-T ${ }_{\mathrm{HR}}$ as to include the residual gross tumor as well as adjacent residual disease $[4,8,9]$.

As evidenced in the initial reports of image-guided $\mathrm{BT}$, the ability to deliver $87 \mathrm{~Gy}$ to the HR-CTV results in improved local control rates above 95\% [10]. This was the first report to suggest that DVH parameters were important for outcomes prediction, and the American Brachytherapy Society (ABS), as well as, the GEC ESTRO currently recommend delivery of $80 \mathrm{~Gy}$ for complete or partially responding tumors $<4 \mathrm{~cm}$ at BT, with dose escalation to 85-90 Gy for non-responding tumors not meeting these criteria $[4,11]$. The importance of dose coverage and optimization made possible by additional interstitial needle placement is therefore best demonstrated by its relation to HR-CTV $D_{90}$. There is currently a lack of prospective randomized data; though, retrospective series provide compelling data for improved target coverage and clinical outcomes when using the hybrid IC/IS approach. Most notably, the RetroEMBRACE study analyzed patients from multiple centers and categorized them into two groups: one intracavitary and one IC/IS BT group. The data demonstrated an improved $D_{90}$ to $\mathrm{HR}-$ CTV with systematic use of IC/IS BT. The reported $\mathrm{D}_{90}$ increased from $83 \pm 14$ Gy to $92 \pm 13$ Gy [12]. Furthermore, there was a significant improvement in local control of $10 \%$ for patients with HR-CTV volume $\geq 30 \mathrm{~cm}^{3}$, with no difference observed for those with HR-CTV $<30 \mathrm{~cm}^{3}$. Our results demonstrated that a HR-CTV $\mathrm{D}_{90} \mathrm{EQD}_{2}>90 \mathrm{~Gy}_{10}$ was associated with improved local control, although this did not reach statistical significance $(\mathrm{HR}=0.25, p=0.19)$.

A separate retrospective single-institution report of outcomes for IC/IS BT in Japan demonstrated good feasibility and a two-year local control rate of $80.2 \%$ [13]. The median HR-CTV volume at initial hybrid BT was $37.1 \mathrm{~cm}^{3}$, with a median HR-CTV $\mathrm{D}_{90}$ of $70.3 \mathrm{~Gy}$ and $\mathrm{V}_{100}$ of $95.9 \%$. Recently, a North American series showed good feasibility of hybrid BT utilizing Vienna and Venezia devices, with a 12-month locoregional control of $80.6 \%$ [14].
Table 5. Univariable logistic regression showing association between organs at risk doses and late genitourinary (GU) or gastrointestinal (GI) toxicities

\begin{tabular}{|c|c|c|}
\hline & $\begin{array}{c}\text { Odds ratio } \\
(95 \% \mathrm{Cl})\end{array}$ & $p$-value \\
\hline \multicolumn{3}{|l|}{ Late GU toxicity } \\
\hline $\mathrm{EQD}_{2}$ bladder $0.1 \mathrm{~cm}^{3}\left(\right.$ per $\left.\mathrm{Gy}_{3}\right)$ & $0.98(0.94,1.04)$ & 0.56 \\
\hline $\mathrm{EQD}_{2}$ bladder $2 \mathrm{~cm}^{3}\left(\right.$ per $\left.\mathrm{Gy}_{3}\right)$ & $0.98(0.90,1.07)$ & 0.68 \\
\hline \multicolumn{3}{|l|}{ Late Gl toxicity } \\
\hline $\mathrm{EQD}_{2}$ bowel $0.1 \mathrm{~cm}^{3}\left(\right.$ per $\left.\mathrm{Gy}_{3}\right)$ & $1.01(0.98,1.05)$ & 0.58 \\
\hline $\mathrm{EQD}_{2}$ bowel $2 \mathrm{~cm}^{3}\left(\right.$ per $\left.\mathrm{Gy}_{3}\right)$ & $1.01(0.95,1.07)$ & 0.73 \\
\hline $\mathrm{EQD}_{2}$ rectum $0.1 \mathrm{~cm}^{3}\left(\right.$ per $\left.\mathrm{Gy}_{3}\right)$ & $1.00(0.95,1.05)$ & 0.87 \\
\hline $\mathrm{EQD}_{2}$ rectum $2 \mathrm{~cm}^{3}\left(\right.$ per $\left.\mathrm{Gy}_{3}\right)$ & $0.98(0.91,1.06)$ & 0.66 \\
\hline $\mathrm{EQD}_{2}$ sigmoid $0.1 \mathrm{~cm}^{3}\left(\right.$ per $\left.\mathrm{Gy}_{3}\right)$ & $1.06(1.01,1.10)$ & 0.015 \\
\hline $\mathrm{EQD}_{2}$ sigmoid $2 \mathrm{~cm}^{3}\left(\right.$ per $\left.\mathrm{Gy}_{3}\right)$ & $1.07(1.00,1.15)$ & 0.059 \\
\hline
\end{tabular}

Here, we demonstrated excellent feasibility and implementation of IGABT for LACC with the use of a hybrid tandem and ovoids applicator with holes for parallel interstitial needle implantation. Our patient population was comprised of mainly FIGO IIB and IIIB cervical cancer patients, with a median HR-CTV volume of $37.87 \mathrm{~cm}^{3}$. Forty percent of our patient population had a median HR-CTV of $>40 \mathrm{~cm}^{3}$ at the time of brachytherapy. Twoyear LC and LRC for stage IIB patients with a mHR-CTV $<40 \mathrm{~cm}^{3}$ were significantly improved, as compared to $\geq 40 \mathrm{~cm}^{3}$ at $100 \%$ and $71.8 \%$, respectively $(p=0.019)$, and $100 \%$ and $56.5 \%$, respectively $(p=0.001)$. However, this trend was not statistically significant for stage IIIB patients. Therefore, confirming the importance of tumor dimensions at hybrid BT versus initial dimensions and FIGO stage group.

Patterns of failure observed in our cohort demonstrated a predisposition for distant failures over local or loco-regional failures. This supports findings that patterns of relapse for cervical cancer treated with IGABT have changed to more distant rather than local failures, as found in an analysis of the RetroEMBRACE cohorts [15]. OAR EQD ${ }_{2}$ doses were comparable with the published median $\mathrm{D}_{2 \mathrm{cc}}$ doses to the bladder, rectum, and sigmoid in the RetroEMBRACE cohort. Our median bladder $2 \mathrm{cc}$ dose was 88.3 Gy compared with $79 \mathrm{~Gy}$, median rectum 2 cc dose was 70 Gy vs. 65 Gy, and median sigmoid 2 cc dose was $70.4 \mathrm{~Gy}$ vs. $65 \mathrm{~Gy}$ [12]. Of note, the sigmoid $0.1 \mathrm{cc}$ $\mathrm{EQD}_{2}$ dose was associated with late GI toxicity, with HR of 1.06 (range, 1.01-1.10) $\left(p=0.015\right.$ ). Bladder $\mathrm{EQD}_{2}$ doses were not associated with late GU toxicity, and neither rectum nor bowel $\mathrm{EQD}_{2}$ doses were associated with late GI toxicity. In the most recent publication from the EMBRACE study group analyzing IC/IS technique, the mean bladder, rectum, and sigmoid $\mathrm{D}_{2 \mathrm{cc}}$ doses for the tandem and ovoids IC/IS group were 79.4, 62.7, and 63.4 Gy, respectively [16]. Of note, our larger median 
HR-CTV volume of $37.87 \mathrm{~cm}^{3}$ may provide some explanation for the slightly higher OARs doses. Specifically, about $30 \%$ of the patients had a bladder $\mathrm{EQD}_{2}>90 \mathrm{~Gy}$. It is worth stating that our OAR dose estimation is based on a uniform dose concept as suggested in GEC ESTRO publications, and not optimized by identifying the most exposed volume of critical organs, as eloquently studied by Frohlich and colleagues $[8,17]$.

Although IC/IS applicators provide improved dose optimization and tumor control compared to IC applicators, it is not known whether the IC/IS approach provides equivalent outcomes when compared to a perineal interstitial implant for larger tumors. In a recent meta-analysis of published series on perineal interstitial BT performed using 3D image guidance, LC was reported at $79 \%$, but ranged from $62-92 \%$ [18]. Sixty percent of patients had stage IIIB disease or higher, though local control or survival was not available on a per stage basis. A single-institution experience with interstitial BT for tumors larger than $30 \mathrm{~cm}^{3}$ demonstrated a two-year local control rate of $77.6 \%$ [19]. Of thirty-seven patients, $65 \%$ had stage IIIB disease or higher ( $49 \%$ stage III, $16 \%$ stage IV), and a median tumor volume of $59 \mathrm{~cm}^{3}$. Despite the large tumor volume, the median HR-CTV $\mathrm{D}_{90}$ was $87.4 \mathrm{~Gy}$. Even having a large percentage of patients $(40 \%)$ with high-volume disease, our clinical outcomes remained comparable to these published local control rates for tumors $>30 \mathrm{~cm}^{3}$ treated with an interstitial implant. We observed twoyear local control rates in stage IIB patients of $71.8 \%$ and $100 \%$ for tumor volumes $\geq 40$ and $<40 \mathrm{~cm}^{3}$, respectively $(p=0.019)$. In stage IIIB patients, the two-year local control rates were $70.7 \%$ and $85.1 \%$ for tumor volumes $\geq 40$ and $<40 \mathrm{~cm}^{3}$, respectively $(p=0.41)$.

In an analysis of 85 cervical tumors treated with IGABT, MRI demonstrating infiltration of the inner, middle, and outer third of the parametrium at diagnosis resulted in a significantly increased risk for residual parametrial disease at brachytherapy ( $88 \%$ vs. $43 \%$ ) [20]. The ability to cover this residual parametrial disease, in addition to consideration of the sidewall and vaginal extent, is the key for hybrid BT selection. Specific applicator selection and referral for brachytherapy may be impacted if initial imaging demonstrates concern for potential residual disease.

IC/IS BT can be performed with either a tandem and ovoids applicator with adaptation for parallel needle placement through the ovoids, or with a tandem and ring applicator with placement through the ring [3, 21]. Recently, a hybrid applicator capable of oblique interstitial needle placement, with an angle of 20 degrees relative to the tandem, was demonstrated to have adequate dose coverage of the distal parametrium and pelvic sidewall, showing local control rates of $76 \%$ and $72 \%$ at 3 and 5 years, respectively [7]. These data suggest that oblique interstitial implantation could represent an alternative to perineal interstitial implant for selected patients with distal residual tumor. In our series, we did not define the extent of involvement of the parametria or sidewall at BT, and therefore it is difficult to compare the outcomes. However, the reported HR-CTV volume across both centers in this series was $71 \mathrm{~cm}^{3}$ compared to our median HR-CTV of $37.9 \mathrm{~cm}^{3}$. Therefore, we likely had a less extensive cohort of tumors at the time of brachytherapy. Furthermore, at our institution, patients with distal extent of disease at BT are typically selected for interstitial implant.

Limitations of this study include its retrospective nature and the use of CT image-guided 3D-based planning, with the use of pre-brachytherapy MRI for response and implant guidance. Though, BT planning was done on CT, there is data to suggest that outcomes are similar when comparing CT- vs. MRI-based contouring, and is performed at other institutions without access to MRI-based simulation [19, 22]. Given the retrospective nature of our analysis, toxicity outcome comparisons may be limited due to observer's bias. There is also a limitation due to short follow-up in a significant proportion of the patients. This is not uncommonly seen at our institution, where significant socio-economic factors have been shown to affect compliance on treatment, which may also contribute to follow-up [23].

\section{Conclusions}

Intracavitary/interstitial brachytherapy using a hybrid tandem and ovoids applicator for LACC provides satisfactory outcomes with excellent local control, overall survival, and modest toxicity profile. Dose coverage assessed by the HR-CTV $D_{90}$ demonstrated a trend towards improved local control with higher dose. However, caution should be exercised when selecting patients for hybrid brachytherapy with a tumor volume $\geq 40 \mathrm{~cm}^{3}$, even though local control outcomes are comparable to published results for interstitial implant.

\section{Disclosure}

The authors report no conflict of interest.

\section{References}

1. Han K, Milosevic M, Fyles A et al. Trends in the utilization of brachytherapy in cervical cancer in the United States. Int J Radiat Oncol Biol Phys 2013; 87: 111-119.

2. Sturdza A, Potter R, Fokdal LU et al. Image guided brachytherapy in locally advanced cervical cancer: Improved pelvic control and survival in RetroEMBRACE, a multicenter cohort study. Radiother Oncol 2016; 120: 428-433.

3. Nomden CN, de Leeuw AA, Moerland MA et al. Clinical use of the Utrecht applicator for combined intracavitary/interstitial brachytherapy treatment in locally advanced cervical cancer. Int J Radiat Oncol Biol Phys 2012; 82: 1424-1430.

4. Haie-Meder C, Potter R, Van Limbergen E et al. Recommendations from Gynaecological (GYN) GEC-ESTRO Working Group (I): concepts and terms in 3D image based 3D treatment planning in cervix cancer brachytherapy with emphasis on MRI assessment of GTV and CTV. Radiother Oncol 2005; 74: 235-245.

5. Pecorelli S, Zigliani L, Odicino F. Revised FIGO staging for carcinoma of the cervix. Int J Gynaecol Obstet 2009; 105: 107-108.

6. Report 89. J ICRU 2016; 13: NP.

7. Mahantshetty U, Sturdza A, Naga ChP et al. Vienna-II ring applicator for distal parametrial/pelvic wall disease in cervical cancer brachytherapy: An experience from two institutions: Clinical feasibility and outcome. Radiother Oncol 2019; 141: $123-129$. 
8. Potter R, Haie-Meder C, Van Limbergen E et al. Recommendations from gynaecological (GYN) GEC ESTRO working group (II): concepts and terms in 3D image-based treatment planning in cervix cancer brachytherapy-3D dose volume parameters and aspects of 3D image-based anatomy, radiation physics, radiobiology. Radiother Oncol 2006; 78: 67-77.

9. Dimopoulos JC, Petrow P, Tanderup K et al. Recommendations from Gynaecological (GYN) GEC-ESTRO Working Group (IV): Basic principles and parameters for MR imaging within the frame of image based adaptive cervix cancer brachytherapy. Radiother Oncol 2012; 103: 113-122.

10. Dimopoulos JC, Lang S, Kirisits C et al. Dose-volume histogram parameters and local tumor control in magnetic resonance image-guided cervical cancer brachytherapy. Int J Radiat Oncol Biol Phys 2009; 75: 56-63.

11. Viswanathan AN, Beriwal S, De Los Santos JF et al. American Brachytherapy Society consensus guidelines for locally advanced carcinoma of the cervix. Part II: high-dose-rate brachytherapy. Brachytherapy 2012; 11: 47-52.

12. Fokdal L, Sturdza A, Mazeron R et al. Image guided adaptive brachytherapy with combined intracavitary and interstitial technique improves the therapeutic ratio in locally advanced cervical cancer: Analysis from the retroEMBRACE study. Radiother Oncol 2016; 120: 434-440.

13. Murakami N, Kobayashi K, Shima S et al. A hybrid technique of intracavitary and interstitial brachytherapy for locally advanced cervical cancer: initial outcomes of a single-institute experience. BMC Cancer 2019; 19: 221.

14. Keller A, Rodríguez-López JL, Patel AK et al. Early outcomes after definitive chemoradiation therapy with Vienna/Venezia hybrid high-dose rate brachytherapy applicators for cervical cancer: A single-institution experience. Brachytherapy 2021; 20: 104-111.

15. Tan LT, Potter R, Sturdza A et al. Change in patterns of failure after image-guided brachytherapy for cervical cancer: analysis from the RetroEMBRACE study. Int J Radiat Oncol Biol Phys 2019; 104: 895-902.

16. Serban M, Kirisits C, de Leeuw A et al. Ring versus ovoids and intracavitary versus intracavitary-interstitial applicators in cervical cancer brachytherapy: results from the EMBRACE I study. Int J Radiat Oncol Biol Phys 2020; 106: 1052-1062.

17. Fröhlich G, Vízkeleti J, Nguyen AN et al. Comparative analysis of image-guided adaptive interstitial brachytherapy and intensity-modulated arc therapy versus conventional treatment techniques in cervical cancer using biological dose summation. J Contemp Brachytherapy 2019; 11: 69-75.

18. Mendez LC, Weiss Y, D'Souza D et al. Three-dimensional-guided perineal-based interstitial brachytherapy in cervical cancer: A systematic review of technique, local control and toxicities. Radiother Oncol 2017; 123: 312-318.

19. Wang C, Raince J, Swamy U et al. Clinical outcomes using image-guided interstitial brachytherapy for definitive cervical cancer patients with high-risk clinical target volumes greater than 30 cc. Brachytherapy 2018; 17: 392-398.

20. Schmid MP, Fidarova E, Pötter R et al. Magnetic resonance imaging for assessment of parametrial tumour spread and regression patterns in adaptive cervix cancer radiotherapy. Acta Oncol 2013; 52: 1384-1390.

21. Kirisits C, Lang S, Dimopoulos J et al. The Vienna applicator for combined intracavitary and interstitial brachytherapy of cervical cancer: design, application, treatment planning, and dosimetric results. Int J Radiat Oncol Biol Phys 2006; 65: 624-630.

22. Kamran SC, Manuel MM, Cho LP et al. Comparison of outcomes for MR-guided versus CT-guided high-dose-rate interstitial brachytherapy in women with locally advanced carcinoma of the cervix. Gynecol Oncol 2017; 145: 284-290.
23. Ohri N, Rapkin BD, Guha C et al. Radiation therapy noncompliance and clinical outcomes in an urban academic cancer center. Int J Radiat Oncol Biol Phys 2016; 95: 563-570. 